\title{
Edema pulmonar no cardiogénico como complicación de sobredosis por consumo de heroína. Reporte de caso
}

\section{Non Cardiogenic Pulmonary Edema as a Complication of Overdose by Heroine Consumption. Case Report}

\author{
Catalina Rodríguez Prada ${ }^{\mathrm{a}}$ \\ Médica residente de Medicina de Urgencias, Pontificia \\ Universidad Javeriana, Bogotá, Colombia \\ Camilo Andrés Jiménez Cruz \\ Médico residente de Medicina de Urgencias, Pontificia \\ Universidad Javeriana, Bogotá, Colombia \\ Juan Carlos Jaimes Gélvez \\ Médico residente de Medicina de Urgencias, Pontificia \\ Universidad Javeriana, Bogotá, Colombia \\ Mario Andrés Enciso Castro \\ Médico residente de Medicina de Urgencias, Pontificia \\ Universidad Javeriana, Bogotá, Colombia \\ Onofre Casa Castro \\ Médico residente de Medicina de Urgencias, Pontificia \\ Universidad Javeriana, Bogotá, Colombia \\ Jorge Andrés SuÁrez Agudelo \\ Médico especialista en Medicina de Urgencias, \\ Pontificia Universidad Javeriana. Médico \\ emergenciólogo, Hospital Universitario San Ignacio, \\ Bogotá, Colombia \\ DANILO SALAZAR \\ Médico especialista en Medicina de Urgencias, Clínica \\ de los Remedios, Cali, Colombia
}

a Autora de correspondencia:
catalinaprada@hotmail.com

Cómo citar: Rodríguez Prada C, Jiménez Cruz CA, Jaimes Gélvez JC, Enciso Castro MA, Casa Castro O, Suárez Agudelo JA, Salazar D. Edema pulmonar no cardiogénico como complicación de sobredosis por consumo de heroína: reporte de caso. Univ. Med. 2020;61(2). https://doi.org/10.11144/Javeriana.umed 61-2.edem.

\section{RESUMEN}

La heroína es una de las drogas ilícitas más utilizadas, potencialmente adictiva, derivada sintéticamente de la morfina, con múltiples efectos adversos secundarios por su uso. El artículo presenta un caso de edema pulmonar asociado a consumo de heroína, como complicación del abuso de esta droga ilícita. La sospecha clínica e identificación clínica, complementada con las imágenes, fueron claves para el diagnóstico. Se recomienda mantener como diagnóstico diferencial el consumo de drogas ilícitas cuando se atiendan patologías del sistema respiratorio.

Palabras clave

dependencia de heroína; edema pulmonar; ventilación pulmonar.

\section{ABSTRACT}

Heroin is one of the most used, potentially addictive drugs, synthetically derived from morphine, with multiple adverse effects in its use. A case of pulmonary edema associated with heroin use is presented, as a 
complication of the abuse of this illicit drug. Clinical suspicion and clinical identification, complementary to the images, are key to the diagnosis. It is recommended to maintain as a differential diagnosis the consumption of illicit drugs when it comes to pathologies of the respiratory system.

Keywords

heroin dependence; pulmonary edema; pulmonary ventilation.

\section{Introducción}

Para poder hablar sobre complicaciones asociadas con sobredosis por heroína, debemos conocer su historia, desde los primeros cultivos de amapola hasta la creación de los opioides sintéticos como la morfina.

La relación del hombre con las propiedades de esta planta está inmersa en la historia misma de la humanidad. Las primeras referencias sobre el uso de la amapola datan del año 3300 a. C., descritas por sumerios en la antigua Mesopotamia. Desde ese entonces se hacía referencia a la amapola como la "planta de la dicha", por lo que esta civilización la usaba por sus propiedades medicinales y recreativas (1).

En el periodo tardío de la China imperial, muchos la usaban como tratamiento para la diarrea y de otros síntomas de enfermedades típicas. Aunque inicialmente fumar opio se consideraba un comportamiento normal de la clase alta, la opinión sobre esta actividad fue cambiando hasta incluso pensar que reflejaba la debilidad de una sociedad consumida por la droga. Por lo anterior, se prohibió desde 1906 hasta 1949, lo que generó una peor enfermedad, pues fue el sustrato necesario para que en 1929 inventaran los opioides semisintéticos, como la morfina y la heroína, y con ello aumentó la ilegalidad en su consumo (2).

De acuerdo con Insight Crime (2013), la dinámica de la producción y exportación de la heroína colombiana es muy variable. En los años noventa, el país era responsable de un $65 \%$ de la heroína en Estados Unidos; sin embargo, en los últimos años, México la ha venido desplazando (1).

$\mathrm{Al}$ revisar los últimos informes sobre el potencial de producción de heroína en Colombia, entre 2013 y 2014 se produjeron entre 1 y 1,5 toneladas; mientras que la producción mundial (con pureza desconocida) se calculó en 506 toneladas, lo que determina que el país participa actualmente con el 0,2\% de la oferta de la heroína en el ámbito mundial (3).

El Informe Mundial de Drogas de 2015 mostró que en la población adulta la prevalencia del consumo de opioides sintéticos es del $0,7 \%$, lo que equivale a 32,4 millones de personas. En Colombia, en 2013 se publicó el Segundo Estudio Nacional de Consumo de Sustancias Psicoactivas, que señaló una prevalencia de consumo del 0,14\%. Para ese año se había registrado que aproximadamente 31.900 personas la habían consumido en algún momento, y en el último año, aproximadamente, 7011 personas la han consumido; sin embargo, en esta misma publicación se observaba un discreto descenso de la prevalencia de 1992 a 2013 (1).

La heroína es una de las drogas ilícitas más utilizadas, con potencial altamente adictivo y su consumo lleva a numerosos desenlaces adversos. Es una droga opiácea, elaborada de la morfina, una sustancia natural que se extrae de la vaina de semillas de amapola. La heroína puede presentarse en polvo blanco o marrón, o una sustancia negra pegajosa (alquitrán negro). Se puede inyectar, inhalar, aspirar o fumar.

Existen complicaciones relacionadas con su consumo, y entre ellas de tipo pulmonar, como edema pulmonar no cardiogénico, neumonitis aspirativa, síndrome de dificultad respiratoria aguda (SDRA), neumonía, absceso pulmonar y embolia pulmonar séptica (4). El primer reporte de edema pulmonar no cardiogénico asociado al consumo de heroína fue hecho por William Osler, en 1880; posterior a esto, los informes han aumentado, porque sigue siendo una complicación grave, ya que aproximadamente el $33 \%$ de las personas que la presentan llegan a requerir ventilación mecánica $(5,6)$.

El uso de la heroína y las complicaciones secundarias a la sobredosis traen consecuencias económicas al sistema de salud colombiano, desde el manejo inicial hasta la rehabilitación del paciente. Por lo anterior, con este artículo queremos que se amplíe el conocimiento sobre las complicaciones del consumo de heroína y, con 
ello, brindar un diagnóstico y tratamiento médico de alta calidad.

\section{Caso clínico}

El caso corresponde a un hombre de 26 años de edad sin aparentes antecedentes, quien fue encontrado por personal de atención prehospitalaria en su domicilio, con alteración del estado de conciencia, cianótico y desaturado, en una condición que orientaba a inyección de drogas ilícitas.

En el momento del ingreso a la sala de reanimación del departamento de emergencias, se encontró a un paciente cianótico con dificultad respiratoria. En el examen neurológico, con miosis pupilar, sin respuesta al llamado, sin obedecer órdenes, con los siguientes signos vitales: FC: 130 latidos/min; FR: 24 respiraciones/min; PA: 90/60 mm Hg y $\mathrm{SatO}_{2}: 60$ $\%$ al aire ambiente. Se administró $\mathrm{FIO}$ al $50 \%$ con sistema Venturi.

Se decidió buscar una posible etiología y descartar complicaciones, por lo que se tomó una tomografía axial computarizada de cráneo simple, en la cual no se observaron alteraciones, y una tomografía computarizada de alta resolución (TACAR) de tórax, donde se observó un patrón compatible con edema pulmonar (figura 1). El paciente estaba en inminencia de falla ventilatoria, sin evidencia de defensa de vía área, por lo que se optó por iniciar medidas de soporte con ventilación mecánica invasiva (VMI) sin requerimiento de soporte vasoactivo.

\section{Figura 1.}

Tomografía de alta resolución de tórax (TACAR).

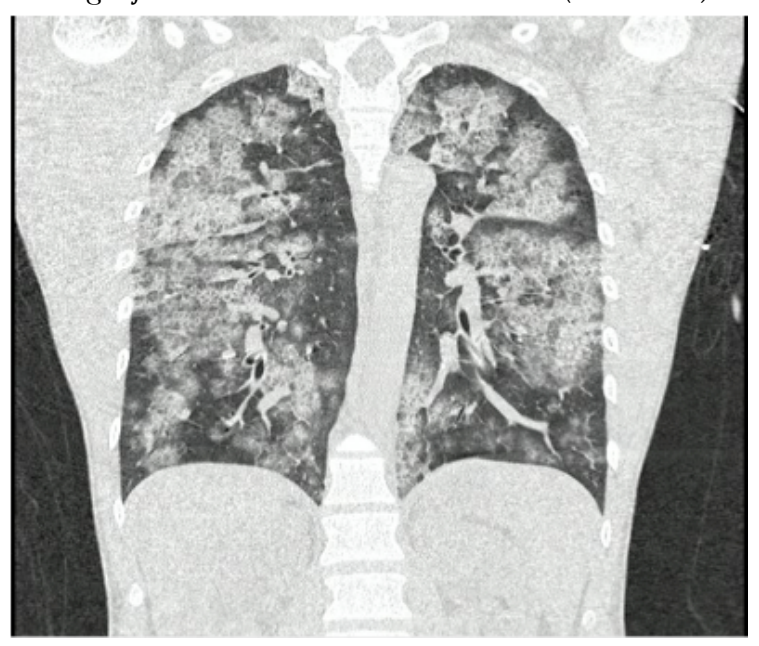

Empedrado en áreas declive de lóbulos superiores e inferiores y en menor proporción con localización centrilobulillar en el lóbulo medio y la língula. Sugestivo de edema pulmonar.

Se le tomaron exámenes paraclínicos, entre ellos radiografía de tórax, que reveló opacidades mixtas de predominio alveolar en ambos campos pulmonares, con tubo endotraqueal a $4 \mathrm{~cm}$ de la carina (figura 2). También gases arteriales después del inicio de la VMI con un $\mathrm{pH}$ de 7,26; $\mathrm{PO}_{2}$ de $70 \mathrm{~mm} \mathrm{Hg} ; \mathrm{PCO}_{2}$ de $35 \mathrm{~mm} \mathrm{Hg}$; $\mathrm{HCO}_{3}$ de $15 \mathrm{BE}-15 ; \mathrm{SO}_{2}$ al $80 \%$; hemograma con leucocitos de 7600; hemoglobina de 18 $\mathrm{g} / \mathrm{dl}$; hematocrito al $54 \%$; neutrófilos al 78 \%; plaquetas: 301.000; creatinina: 2,61 mg/dl. Electrolitos: sodio de $135 \mathrm{mmol} / \mathrm{l}$; potasio de 5,3 $\mathrm{mmol} / \mathrm{l}$; cloro de $104 \mathrm{mmol} / \mathrm{l}$. 


\section{Figura 2.}

Radiografía de tórax.

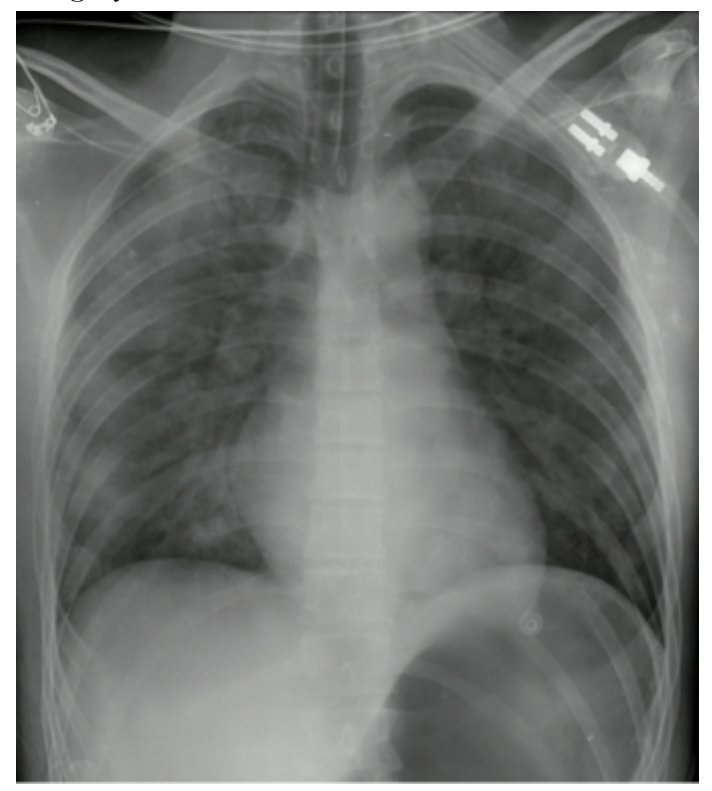

Opacidades mixtas de predominio alveolar en ambos campos pulmonares, con tubo endotraqueal a $4 \mathrm{~cm}$ de la carina.

El panel toxicológico de drogas de abuso en la orina con benzodiacepinas dio menor a $18 \mathrm{ng} / \mathrm{ml}$ (punto de corte hasta $200 \mathrm{ng} / \mathrm{ml}$ ); barbitúricos, menores a $25 \mathrm{ng} / \mathrm{ml}$ (punto de corte hasta $200 \mathrm{ng} /$ $\mathrm{ml}$ ); cannabinoides, mayores a $200 \mathrm{ng} / \mathrm{ml}$ (punto de corte $50 \mathrm{ng} / \mathrm{ml}$ ); cocaína, inferior a $40 \mathrm{ng} /$ $\mathrm{ml}$ (punto de corte hasta $150 \mathrm{ng} / \mathrm{ml}$ ); opiáceos, superiores a $1000 \mathrm{ng} / \mathrm{ml}$ (punto de corte hasta $300 \mathrm{ng} / \mathrm{ml}$ ); y anfetaminas, inferiores a $100 \mathrm{ng} /$ $\mathrm{ml}$ (punto de corte hasta $500 \mathrm{ng} / \mathrm{ml}$ ). Por todo lo anterior, se consideró que el paciente cursaba con edema pulmonar no cardiogénico por sobredosis de heroína y, adicionalmente, con lesión renal aguda.

Al siguiente día se solicitó la toma de péptido natriurético (BNP), que estaba en 862, y creatinafosfocinasa (CPK), que estaba en $4395 \mathrm{U} / \mathrm{L}$ (39-308). Por ello, se consideró que el paciente cursaba con un proceso de rabdomiólisis, configurando otra complicación asociada a la sobredosis de heroína, y se realizó el ajuste del manejo con lactato de Ringer, con un bolo inicial de $20 \mathrm{~cm}^{3} / \mathrm{kg}$, y continuaba con infusión a $1,5 \mathrm{~cm}^{3} / \mathrm{kg} / \mathrm{h}$. Se le tomó ecocardiograma en el cual no se evidenciaron trastornos de la contractilidad, con una fracción de eyección del ventrículo izquierdo del $45 \%$.

Se le continuó el manejo al paciente en la unidad de cuidados intensivos durante, aproximadamente, 9 días más. Posterior a esto, salió al servicio de hospitalización sin dificultad respiratoria y sin requerimiento de oxígeno domiciliario (figura 3). Luego, el manejo estuvo a cargo del grupo de psiquiatría, al cual el paciente le comentó que desde la adolescencia había consumido múltiples sustancias psicoactivas. Se encontraron rasgos mal adaptativos de la personalidad. Durante un día más estuvo hospitalizado, sin síntomas de abstinencia y en fase contemplativa de ingreso a un programa de rehabilitación, al que actualmente se encuentra asistiendo.

\section{Figura 3.}

Radiografía de tórax.

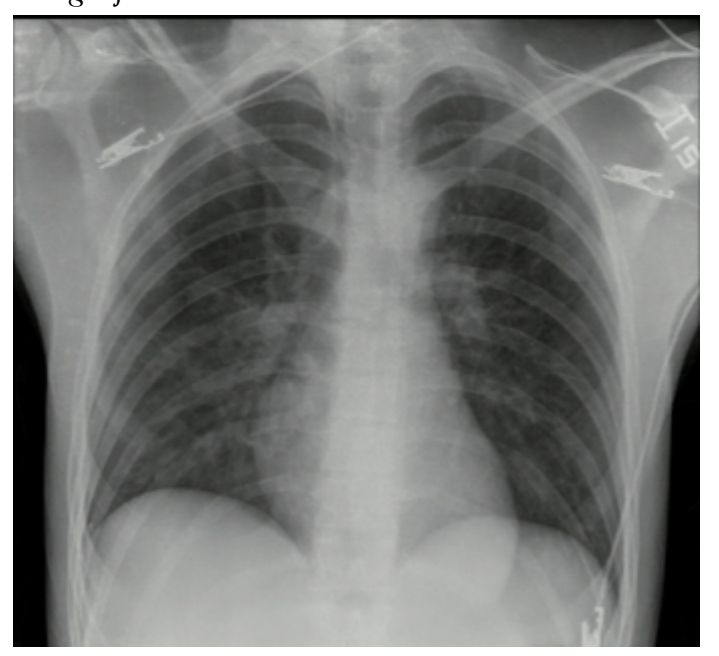

Pequeños infiltrados alveolares difusos, comparados con estudios previos en disminución.

\section{Discusión}

Los fármacos opioides pueden ser naturales, derivados del opio o sintéticos. Presentan varias acciones farmacológicas, como analgesia, sedación y sueño. Su acción depende de un sistema de opioides endógenos que derivan de tres polipéptidos precursores: la proencefalina, de la que derivan las encefalinas; la prodinorfina, de la que se sintetizan las dinorfinas y las neoendorfinas, y la propiomelanocortina, de la 
que derivan las $\beta$-endorfinas. Tienen, además, subtipos de receptores acoplados a proteínas $G$ $(\mu 1,2 ; \kappa 1,2,3 ; \Delta 1,2 ; \Sigma$ y $\varepsilon)$ con amplia distribución en el sistema nervioso central y en el periférico (7). Activan las vías dopaminérgicas mesolímbicas y mesocorticales, especialmente en el área ventral tegmental y el núcleo Accumbens e inhiben las neuronas que tienen acción inhibitoria sobre neuronas dopaminérgicas en el área ventral tegmental o activando los receptores opioides presinápticos, lo que facilita la liberación de dopamina (7).

La heroína (diacetilmorfina) es el opiáceo que actúa más rápido en sistema nervioso central. En el cerebro actúa en 15-30 segundos provocando una sensación de subida o flash, que consiste en una sensación subjetiva muy agradable de calor con goce intenso; posteriormente, euforia y relajación, que tiene una duración de 5-15 minutos, seguida del llamado flooding o profunda sensación de bienestar y plenitud, lo que facilita su adicción y dependencia $(2,7)$. Presenta una tríada donde hay evidencia de miosis, alteración en el patrón respiratorio y alteración del estado de conciencia. Lo anterior lo evidenciamos como el estado clínico de ingreso de nuestro paciente en el caso clínico.

Al revisar la literatura sobre el tema, encontramos que la definición de edema pulmonar no cardiogéncio asociado al consumo de heroína está descrita como un síndrome donde el paciente desarrolla hipoxia $\left(\mathrm{SatO}_{2}<90 \%\right)$ en menos de 24 horas de la última sobredosis de heroína, acompañado de evidencia radiológica de infiltrados difusos, que descartan otras causas, y que se resuelven en un periodo máximo de 48 horas (8).

A pesar de que los primeros casos se describieron aproximadamente hace 100 años, en cuanto a la fisiopatología del edema pulmonar no cardiogénico, en realidad no ha sido completamente identificada, sobre todo porque al hablar de complicaciones, la principal es la muerte, y ocupa apenas un $10 \%$ de los pacientes con sobredosis por heroína. Ello nos lleva a pensar que el edema pulmonar no cardiogénico hace parte de la evolución natural de la muerte por sobredosis de heroína. Actualmente, la literatura describe que primero existe un daño por hipoxia en los neumocitos, que altera la permeabilidad alveolar (9). Lo anterior se ha evidenciado en el análisis del líquido pleural de los pacientes, pues se ha encontrado una mayor concentración de proteínas, en comparación con los pacientes con edema pulmonar de origen cardiogénico, lo cual demuestra que el daño es en la barrera de difusión y no una disfunción en el miocardio $(8,9)$.

También se ha demostrado que este tipo de edema pulmonar puede estar relacionado con una liberación exagerada de histamina y se ha encontrado que los opiáceos causan su liberación sistémica y local. Se ha demostrado que la histamina aumenta el flujo linfático pulmonar y la permeabilidad capilar $(8,9)$.

Por último, hay una hipótesis de que el edema pulmonar podría ser secundario al uso de naloxona como medida para revertir la sobredosis por heroína, secundario a un aumento en la liberación de catecolaminas a nivel sistémico, que producen aturdimiento cardiaco y disfunción miocárdica (9); sin embargo, algo que hace interesante nuestro reporte de caso es que el edema pulmonar no cardiogénico en el paciente se desarrolló sin administración de naloxona.

Realmente, no se han encontraron factores de riesgo que faciliten el desarrollo de esta complicación; pero ocurren con mayor frecuencia en pacientes que ya hayan presentado sobredosis o que tengan difícil acceso a centros prestadores de salud y al no uso de naloxona (1).

El tratamiento son medidas de soporte, y según las recomendaciones de la Organización Mundial de la Salud (OMS), siempre que se sospeche intoxicación por opioides se debe usar naloxona. La dosis proviene de la guía de práctica clínica basada en la evidencia de la OMS, de 2014, para el manejo comunitario de la sobredosis por opioides (10), y de la actualización de las guías de la American Heart Association para reanimación cardiopulmonar y atención cardiovascular de emergencia, de 2015 (11), donde se recomienda administración inicial de 0,4 mg hasta un máximo de $2 \mathrm{mg}$ de acuerdo con la respuesta clínica del paciente. No obstante, el paciente cursaba con un edema pulmonar no cardiogénico, por lo que 
el manejo inicial consistió en corrección de la hipoxemia, y si hubiera habido deterioro clínico, iniciar manejo con ventilación mecánica invasiva (12).

Sin embargo, no solo se debe vigilar el sistema respiratorio, como lo describimos antes, pues la heroína, por sí sola, puede producir rabdomiólisis, lo que lleva al desarrollo de una posible insuficiencia renal. Tal es el motivo por el cual hay que vigilar la CPK total y la función renal; además, siempre es importante descartar daño miocárdico, por lo que hay que tomar BNP y ecocardiograma, que en el caso de nuestro paciente cursaba con rabdomiólisis y con lesión miocárdica, evidenciado por la CPK total elevada y el BNP elevado.

Actualmente no existe una guía que aborde el manejo integral de las complicaciones asociadas a sobredosis. Al revisar los lineamientos para el Protocolo de Manejo de la Sobredosis de Opioides en la Comunidad (13), la Guía clínica para el tratamiento de la dependencia de opiáceos (14) y la Guía para el manejo de emergencias toxicológicas colombianas (15) no se encuentra un consenso sobre el manejo de este tipo de complicaciones, lo que nos invita a desarrollar una guía de manejo institucional o nacional, ya que, como observamos, una adecuada intervención permite que se logre un buen pronóstico en la evolución del paciente.

Por último, esta presentación de caso nos ayuda a reflexionar respecto a que si bien el consumo de heroína en nuestro país aún no se puede comparar con otros países, tenemos que estar atentos a la crisis que se vive a nivel mundial con su consumo y las complicaciones que genera, cuando nos enfrentemos a pacientes con ingesta de sustancias psicoactivas.

\section{Referencias}

1. Ministerio de Justicia y del Derecho, Observatorio de Drogas de Colombia. La heroína en Colombia: producción, uso e impacto en la salud pública. Análisis de la evidencia y recomendaciones de política. Bogotá: ODC; 2015.

2. Dikotter F, Laamann L, Zhou X. Narcotic culture: a history of drugs in China. Chicago: The University of Chicago Press; 2004.

3. Alcaldía Mayor de Bogotá. Vigilancia epidemiológica del abuso sustancias psicoactivas a través del subsistema-VESPA en el Distrito Capital. Bogotá; 2012.

4. Toro Ocampo JH, Álvarez Gómez ML. Complicaciones del uso de la heroína parenteral: artículo de revisión. Med UPB. 2017;36(2):146-52.

5. Sterrett C, Brownfield J, Korn CS, Hollinger M, Henderson SO. Patterns of presentation in heroin overdose resulting in pulmonary edema. Am J Emerg Med. 2003;21(1):32-4.

6. Morrison WJ, Wetherill S, Zyroff J. The acute pulmonary edema of heroin intoxication. Radiology [Internet]. 1970;97 (2):347-51. https://doi.org/10. 1148/97.2.347

7. Yaksh T, Wallece M. Opioides, analgesia y control del dolor. En: Goodman y Gilman: las bases farmacológicas de la terapéutica. 12a ed. México: McGraw Hill; 2011. p. 481-7.

8. Sporer KA, Dorn E. Heroinrelated noncardiogenic pulmonary edema: A case series. Chest. 2001;120(5):1628-32.

9. Bazoukis G, Spiliopoulou A, Mourouzis K, Grigoropoulou P, Yalouris A. Non-cardiogenic pulmonary edema, rhabdomyolysis and myocardial injury following heroin inhalation: A case report. Hippokratia. 2016;20(1):84-7.

10. World Health Organization. Community management of opioid overdose. Geneva; 2014. Disponible 
en: https://apps.who.int/iris/handle/10 $665 / 137462$

11. Neumar RW, Shuster M, Callaway CW, Gent LM, Atkins $\mathrm{DL}$, Bhanji $\mathrm{F}$ et al. American Heart Association guidelines update for cardiopulmonary resuscitation and emergency cardiovascular care. Circulation. 2015;132(18 sup).

12. Farney A. Opioid related non-cardiogenic pulmonary edema [internet]. EMS1 [blog] 2018 nov. Disponible en: https://www.ems1.com/ drugs/articles/opioid-related-non-cardi ogenic-pulmonary-edema-gGMfOuaIe yJ3X1R9/

13. Ministerio de Salud de Colombia. Lineamientos para el protocolo de manejo de la sobredosis de opioides en la comunidad. Bogotá; 2017. Disponible en: https:/www.minsalud.gov.co/sites/ rid/Lists/BibliotecaDigital/RIDE/VS/P P/ENT/protocolo-naloxona-final-2.pdf

14. Fernández Miranda J, Pereiro Gómez C. Guía para el tratamiento de la dependencia de opiáceos. Adicciones: Revista de Socidrogalcohol. 2007;19(extra 2):7-118.

15. Ministerio de Salud y Protección Social de Colombia, Organización Panamericana de la Salud y Organización Mundial de la Salud. Guía para el manejo de emergencias toxicológicas [internet]. Bogotá; 2017. Disponible en: https://www.minsalud.gov.co/sites/ rid/Lists/BibliotecaDigital/RIDE/DE/G T/guias-manejo-emergencias-toxicolog icas-outpout.pdf 